\title{
Multi-time-scale analysis of fatigue crack growth on interfaces via cohesive-zone models
}

\author{
Giulio Alfano
}

School of Engineering and Design, Brunel University, Kingston Lane, UB8 3PH, Uxbridge, UK e-mail: giulio.alfano@brunel.ac.uk

Keywords: Multi-scale methods; cyclic loading; interface elements; hysteretic response; sub-critical damage.

\begin{abstract}
The paper describes a new non-linear finite-element formulation to analyse fatigue debonding or delamination, along predefined interfaces, which is multi-scale in time. At the small time-scale level cyclic loading and the related oscillating response is considered in an explicit way, whereas at the large time-scale level both the real loading actions and the related response in terms of displacement and stress fields are replaced with 'minimum' and 'maximum' functions over the time of the analysis, which also implies doubling the degrees of freedom of the finite-element model. A cohesive-zone model capable of simulating sub-critical damage growth and hysteretic local response is used on the interface. With a conventional cycleby-cycle incremental procedure the analysis would require a number of increments significantly higher than the number of cycles, and would be therefore unfeasible for most industrial applications. Instead, with the developed multi-time-scale method the cycle-by-cycle time integration is transferred from the structural level to the local, integration-point level whereby the time step can be, and in fact should better be, much larger than the frequency of the applied actions. The consequent significant saving in terms of computational cost largely offsets the shortcoming of having to double the degrees of freedom of the model and makes the analysis not only feasible but relatively inexpensive in many cases, while retaining excellent accuracy as showed by the presented numerical results.
\end{abstract}

\section{INTRODUCTION}

The extreme difficulty in modelling and analysing fatigue-crack propagation in structures has led to design procedures mainly based on time-consuming experimental fatigue tests, whose results are correlated using semi-empirical models derived from Paris' law [1-3]. While for conventional materials like steel and aluminium alloys a great amount of experimental data are available on fatigue crack propagation, these procedures become extremely expensive when applied to the design of new materials, for which little previous experience is available, such as reinforced polymer composites or 
adhesives employed in adhesive joints. More importantly, it is now widely recognised that fatigue delamination in non-metals involves different physical mechanisms, which are often not well captured by Paris' law. The constant load amplitude and ratio used in the experimental testing represents a further limitation of the predictive capabilities of the above design approach. This is because the damage accumulation laws based on Miner's rule often do not provide satisfactory approximation of the fatigue life [2]. Furthermore, Paris' law assumes the existence of an initial crack and, therefore, is not able to simulate crack initiation.

For the above reasons, more predictive numerical models can lead to significant saving of time and money, and cohesive-zone models (CZMs) are widely considered as a potentially valid alternative. The response of a CZM in the case of alternating loading depends on the local response to unloading and re-loading. Most CZMs proposed in the literature assume elastic damage only, the secant straight line to the origin being used during unloading, see $[4,5]$ among many others Referring to a single-mode case, and denoting by $\sigma$ and $\delta$ the interface stress and the relative displacement, in these cases the same line of the $\sigma-\delta$ law is followed both during unloading and during re-loading below the maximum previously attained value. This implies that, if cyclic loading with constant amplitude is applied, at each point of the interface the response stabilises immediately after the first cycle, no energy is dissipated and no fatigue crack growth is predicted for cyclic loading (Figure 1.a).

To overcome this problem and predict fatigue crack growth, several CZMs have recently been proposed [6-16]. In fact, all these models use an approach very similar to that used much earlier by Marigo [17]. They share the principle that to simulate the degradation of the interface for alternating values of the relative displacements the model must entail different unloading and re-loading paths, in such a way that (a) a 'hysteresis loop' is obtained and (b) for a relative displacement cyclically oscillating between two given values, the corresponding traction is progressively reduced (Figure 1.b). With these models, small increase in damage for each loading-unloading cycle, which accumulates over time, is obtained even if the relative displacement does not exceed the maximum previously attained value. Following Roe and Siegmund [8], this type of damage growth will be referred to as 'sub-critical'. Instead, if the relative displacement exceeds the maximum previously attained value, for all of these models damage increases instantaneously to values only determined by the increase in the relative displacement itself. This type of damage growth will be indicated as 'monotonic'. A conceptually similar approach to simulate low-cycle fatigue was used by Yang et al. [18].

The different choices for the loading and unloading curves so far proposed in the literature [6-18] reflect the fact that the underlying physics of the problem is still unclear. In fact most of the the models are essentially derived with a purely phenomenological approach, a notable exception being the approach followed in Refs. $[12,14,15]$ where physically sound variational arguments are used to derive CZMs that capture fatigue damage starting from expressions of the surface energy of the Dugdale-Barenblatt (i.e. cohesive) type. Instead, the authors show that with a Griffith type of surface energy no crack propagation can be obtained under cyclic loading. In the limit when the length of the process zone and the energy release rate tend to zero, the authors also demonstrate that the derived fatigue laws tend to Paris' law, different Paris' law constants being 

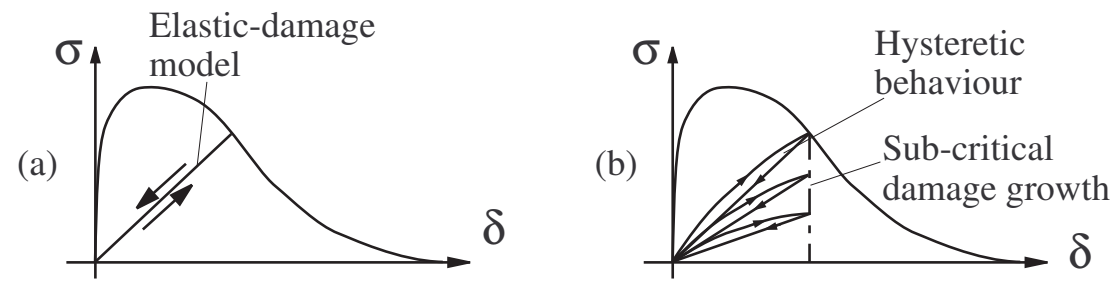

Figure 1: Cohesive-zone models: (a) loading-unloading lines for elastic-damage models; (b) hysteretic models with sub-critical damage growth.

obtained with different forms of surface energy.

The variational approach provides a physically well justified and therefore appealing framework whereby all the above mentioned CZMs [6-18] represent a promising stepforward towards a more predictive strategy in the analysis of fatigue delamination or debonding. On the other hand, apart from more work needed for deeper understanding of their link with the micromechanics of the problem and for their experimental validation and calibration, their main disadvantage is that the simulation of high-cycle fatigue for real-life engineering problems would typically require the analysis of at least many thousands of loading cycles, each of them requiring a certain number of load increments, which is typically not feasible.

An alternative way to use CZMs for the analysis of fatigue crack growth which, instead, is suited to simulate high-cycle fatigue has been presented by Violeau [19]. The main idea was to follow an approach conceptually similar to that used by Peerlings et al. [20] in the context of continuum fatigue-damage models and, therefore, to replace the cyclic loading history with its envelope and consider the number of cycles as a 'real-valued' variable. In fact, with the further assumption of rate-independence of the material response, in this approach the number of cycles can be assumed as a pseudotime variable. A damage variable $D$ is then introduced to simulate degradation of the interface and its rate of change is decomposed in the sum of two terms, so that it results $\dot{D}=\dot{D}_{s}+\dot{D}_{f}$. The first term, $\dot{D}_{s}$, also denoted 'static' damage (rate) in Ref. [21], represents the rate of change which would be obtained under the current load value, if the (envelope) load was applied in a monotonic way; the second term, $\dot{D}_{f}$, also called 'fatigue' damage (rate) in Ref. [21], represents the rate of change which would be obtained under the same load value, but in the hypothesis that the load was applied cyclically, with constant amplitude and a maximum value equal to the load value itself. In the simulations it was always assumed that the minimum value of the cyclic loading was zero, implying a load ratio $R=0$, because in typical non-linear, incremental finite-element analyses one loading function only can be considered. Violeau's formulation was later validated experimentally by Robinson et al. [21]. An attempt to remove the limitation to the case $R=0$ was later made by Muñoz et al. [22] who introduce a prefixed load ratio $R$, with some assumption on the related response.

Starting from the same additive decomposition proposed by Violeau [19], Turon et al. [23] proposed an alternative strategy for high-cycle fatigue that links together the fracture-mechanics and the damage-mechanics approaches essentially translating Paris' 
law into damage evolution laws for a cohesive-zone model. One advantage of their model is that it incorporates both a fatigue threshold and a sensitivity to the load ratio. One disadvantage is that the method requires an analytical estimate of the size of the process zone. May and Hallet [24] also pointed out that one limitation of this approach is the relatively inaccurate prediction of fatigue-damage initiation in absence of initial cracks, which somehow undermines one of the main advantages entailed by the use of cohesive-zone models. To improve the model in this respect they introduced an additional damage evolution mechanism which models damage initiation with a phenomenological approach based on S-N curves.

In the author's opinion, despite the above discussed issues and others shortcomings including the difficulty of robust and reliable identification of mixed-mode dependent model parameters, the method proposed in Ref. [23] and other strategies proposed later along conceptually similar lines [24-28] currently represent the most practically convenient approach for industrial applications. However, with a view to future further developments of formulations, such as those in Refs. [12,14, 15], where a CZMs capturing fatigue crack initiation and propagation can be derived based on physically sound principles (if not 'first principles'), it is still desirable to derive effective numerical methods which allow using such models for high-cycle fatigue.

Furthermore, it is also desirable to have a formulation in which the (possible variable) load ratio is not given as part of the input data of the problem. This is because when the bulk material behaves as linearly elastic, the ratio between maximum and minimum relative displacements at the interface are equal to the applied load ratio. However, for non-linear behaviour of the bulk material this is typically no longer the case.

Therefore, in this paper a new computational method is developed which is based on the same idea as in Violeau's work of replacing the cyclic loading with its maximum and minimum envelopes, but goes one step further because it removes the limitation on the load ratio, does not assume the additive decomposition and provides a basis for a quite more general solution strategy.

The underlying principle for the proposed method is that fatigue is a multi-scale phenomenon in space and in time. This principle is not new and is indeed stated by Fish and Oskay [29], who introduce a decomposition of all response fields in the sum of a 'macro-chronological' (homogenised in time) part and 'micro-chronological' (oscillatory) part, in the framework of a general theory based on the introduction of 'almostperiodical' functions. This is applied to the analysis of crack growth using continuum damage mechanics and the Gurson-Tvergaard-Needleman model, the advance of crack being simulated eliminating elements reaching a threshold of damage.

The method proposed in this paper is different from Fish and Oskay's strategy. It is based on a CZM, which is an implicit way to resolve the multi-scale nature of the problem in space, so that the novelty of the formulation consists of making explicit use of the multi-scale nature of the problem in time. At the small time-scale level the cyclic loading history and the related oscillating response are considered in an explicit way, whereas at the large-scale level both the real loading actions and the related response in terms of displacement and stress fields are replaced with suitably defined 'minimum' and 'maximum' functions over the time of the analysis, which also implies doubling the degrees of freedom of the finite-element model. As already observed, with a conventional cycle-by-cycle incremental procedure the analysis would require a very high 
number of increments, which would be unfeasible for most industrial applications. Instead, with the developed multi-time-scale method the cycle-by-cycle time integration is transferred from the structural level to the local, integration-point level whereby the time step can be, and in fact should better be, much larger than the frequency of the applied actions. The consequent significant saving in terms of computational cost largely offsets the shortcoming of having to double the degrees of freedom of the model and makes the analysis not only feasible but relatively inexpensive in most cases. Despite some assumptions made on the relative-displacement variation in time, the presented numerical results show that the developed multi-time-scale method provides excellent accuracy both for a case of constant amplitude and when amplitude and mean values are varied.

The detailed, cycle-by-cycle time integration at the integration point is made using a CZM which simulates hysteretic response and sub-critical damage growth, similar to those proposed in Refs. [6-9,11], but simpler in the formulation and implementation. The CZM model admits an approximated closed-form time-integration of the smallscale problem at each integration point. In this first formulation of the method the treatment is limited to the single-mode case and numerical results are presented for the analysis of a double-cantilever-beam (DCB) made of two aluminium plates joined by an epoxy adhesive and tested under cyclic prescribed displacement in mode-I.

The structure of the paper is as follows. In Section 2 the proposed CZM, which simulates hysteretic response and sub-critical damage growth, is presented. The multitime-scale solution strategy is first outlined in its general form in Section 3 and then specialised to the proposed CZM in Section 4. In Section 5 numerical results and their comparison with experimental data are provided. Finally, conclusions are drawn in Section 6, where future lines of research to fully develop the potential of the proposed method are suggested.

\section{COHESIVE-ZONE MODEL}

This section describes the new, proposed cohesive-zone model, which simulates both sub-critical and monotonic damage growth, developed along the lines of similar work $[6-11,13]$. This model is later used in the framework of the multi-time-scale solution scheme presented in Sections 3 and 4.

The formulation of the cohesive-zone model is restricted to a mode-I problem, which is the case considered in the numerical applications. A damage variable $D$ is introduced, and the relationship between the relative displacement $\delta$ and the interface stress $\sigma$ is provided by the classic damage-mechanics law:

$$
\sigma=(1-D) K<\delta>_{+}+K<\delta>_{-}
$$

where $K$ denotes the initial stiffness, typically high enough to well capture the initial undamaged response [4]. Symbols $\langle\delta\rangle_{+}$and $\langle\delta\rangle_{-}$denote the positive and negative parts of the relative displacement $\delta$, whereby in compression the high stiffness is used regardless of the damage, entailing negligible material interpenetration.

In the case of monotonic increase of the relative displacement the value of the dam- 
age variable is given by:

$$
D=\left\{\begin{array}{llcl}
0 & \text { if } & \delta \leq \delta_{0} \text { or } \delta \geq \delta_{c} & \text { (no damage or full damage) } \\
\frac{\delta-\delta_{0}}{\beta \delta} & \text { if } & \delta_{0}<\delta<\delta_{c} & \text { (damage growth) }
\end{array}\right.
$$

where $\delta_{0}$ is the value of the relative displacement below which no damage occurs, $\delta_{c}$ is the critical relative displacement value beyond which cohesion is completely lost and $\beta=1-\left(\delta_{0} / \delta_{c}\right)$. The above damage law results in the widely used bi-linear tractionseparation law depicted in Figure 2.a [4]. As independent parameters of this law one can choose the interface fracture energy $G_{c}$, which is the area under the bilinear curve, the interface strength $\sigma_{0}$ and the ratio $\beta$ between $\delta_{0}$ and $\delta_{c}$. These other parameters are then obtained by:

$$
\delta_{c}=\frac{2 G_{c}}{\sigma_{0}} \quad \delta_{0}=(1-\beta) \delta_{c} \quad K=\frac{\sigma_{0}}{\delta_{0}}=\left(\frac{1}{1-\beta}\right) \frac{\sigma_{0}}{\delta_{c}}
$$

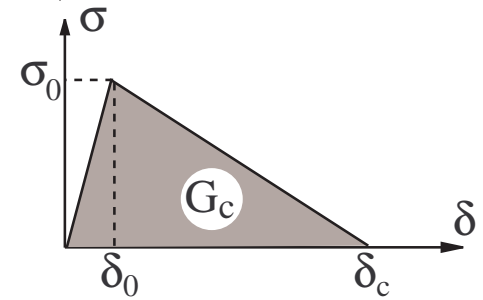

(a)

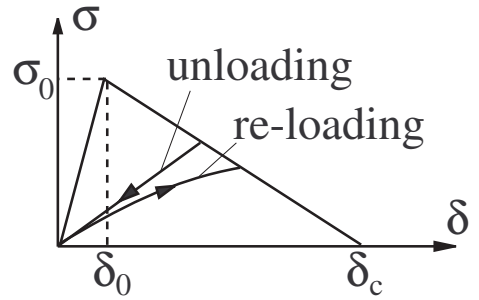

(b)

Figure 2: (a) bi-linear traction-separation law for the case of a monotonic increasing relative displacement; (b) unloading/re-loading paths.

In the general case when the displacement can increase or decrease at different times, the following variable is first introduced as a function of time $t$ :

$$
\delta^{*}=\delta^{*}(t)=\max \left\{\max _{\tau \leq t} \delta(\tau), \frac{\delta_{0}}{1-\beta D(t)}\right\}
$$

Hence, either $\delta^{*}$ is the actual maximum previously attained relative displacement or, during sub-critical damage growth, it is equal to the maximum between the latter and the relative displacement that would correspond to the current damage if the process was monotonic, as will be better clarified below.

The damage evolution is then given by the following relationship: 


$$
\frac{d D}{d \delta}=\left\{\begin{array}{lll}
0 & \text { if } \quad \delta<0 \quad \text { (compression) } \\
0 & \text { if } \quad \delta^{*} \leq \delta_{0} \text { or } \delta^{*} \geq \delta_{c} \quad \text { (no damage or full damage) } \\
\frac{\delta_{0}}{\beta \delta^{2}} & \text { if } \quad \delta_{0}<\delta^{*}<\delta_{c} \text { and } \delta=\delta^{*} \text { and } \dot{\delta}>0 \quad \text { (monotonic damage growth) } \\
0 & \text { if } \quad \delta_{0}<\delta^{*}<\delta_{c} \text { and } 0<\delta \leq \delta^{*} \text { and } \dot{\delta} \leq 0 \quad \text { (unloading) } \\
\gamma D & \text { if } \quad \delta_{0}<\delta^{*}<\delta_{c} \text { and } 0<\delta<\delta^{*} \text { and } \dot{\delta}>0 \quad \text { (re-loading) }
\end{array}\right.
$$

where $\gamma$ is a material parameter governing the rate of sub-critical damage growth.

The cases of compression $\delta<0$, when no damage has occurred yet $\left(\delta^{*} \leq \delta_{0}\right)$ or when complete damage has occurred $\left(\delta^{*} \geq \delta_{c}\right)$ are straightforward.

The case of 'monotonic damage growth' is obtained when the relative displacement is between $\delta_{0}$ and $\delta_{c}$ and increases above $\delta^{*}$. In this case $\delta^{*}$ also increases being equal to $\delta$, and the bi-linear traction-separation law of Figure 2.a is followed. The derivative of $D$ with respect to $\delta$ is therefore simply obtained by differentiating Equation (2) 2 .

In the case of unloading $(\dot{\delta} \leq 0)$, with $0<\delta \leq \delta^{*}$, and when $D$ is neither 0 nor 1 (equivalent to $\delta_{0}<\delta^{*}<\delta_{c}$ ), no damage growth is assumed, as expressed by Equation $(5)_{4}$.

Finally, during reloading $(\dot{\delta}>0)$ after unloading, but in the sub-critical case whereby $0<\delta<\delta^{*}$, and when $D$ is neither 0 nor 1 , the damage is assumed to increase following Equation $(5)_{5}$.

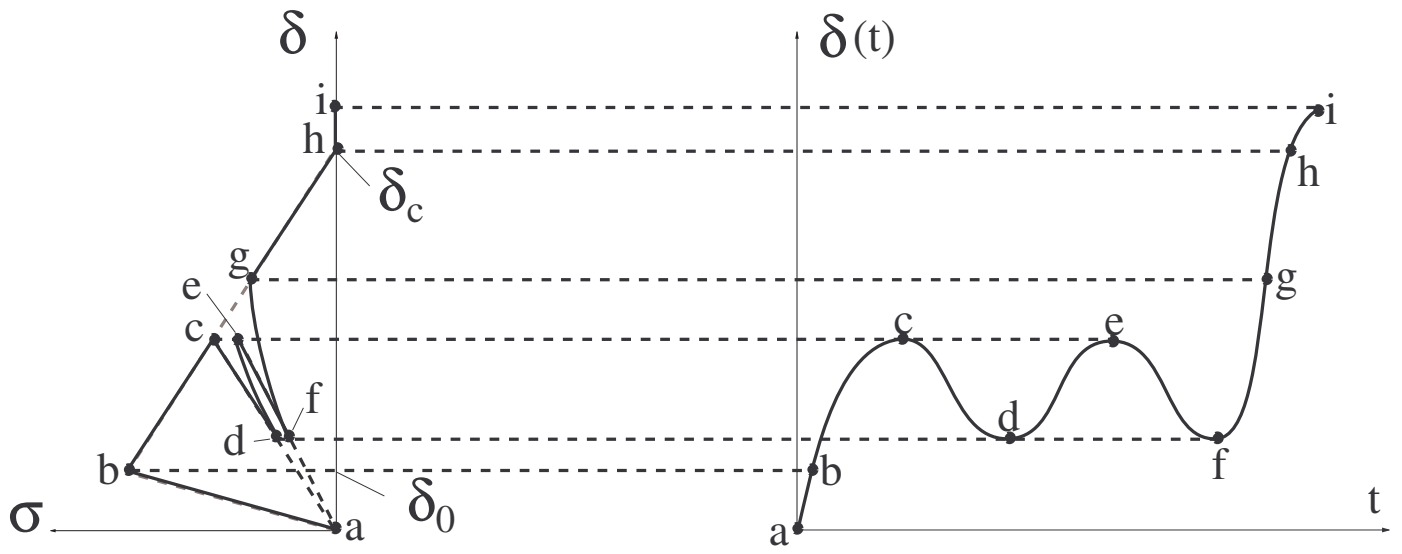

Figure 3: Cohesive-zone model developed and used in this work: (a) interface stress vs relative displacement caused by a (b) prescribed relative-displacement history.

In order to better explain the meaning of Equations (4) and (5), Figure 3.a reports the 'traction-relative displacement' plot resulting from the prescribed relative-displacement 
history of Figure 3.b, for an initially undamaged interface, if the proposed CZM is used. The response on each branch of the curve is explained as follows.

Starting from $D=0$, between points $a$ and $b$ it is $0<\delta=\delta^{*} \leq \delta_{0}$, resulting in no damage growth in accordance with Equation $(5)_{2}$.

Between points $b$ and $c$ monotonic damage growth occurs, because $\delta_{0}<\delta=\delta^{*}<\delta_{c}$ and $\dot{\delta}>0$, following Equation $(5)_{3}$ and resulting in a constant negative slope.

Between points $c$ and $d$ it results $\dot{\delta}<0$. Hence, $\delta<\delta^{*}, \delta_{0}<\delta^{*}<\delta_{c}$ and Equation $(5)_{4}$ applies resulting in unloading with no damage growth.

From point $d$ to point $e$ re-loading occurs because $\dot{\delta}>0$ and $\delta<\delta^{*}$ so that Equation $(5)_{5}$ applies and sub-critical damage growth occurs. Furthermore, because of Equation (4), the value of $\delta^{*}$ actually increases in this part. To verify this, let us observe that up to point $c$ only monotonic damage growth was found whereby Equation $(2)_{2}$ yields the following expression for $D\left(t_{c}\right)$ :

$$
D\left(t_{c}\right)=\frac{\delta\left(t_{c}\right)-\delta_{0}}{\beta \delta\left(t_{c}\right)}
$$

Rearranging, and since $\delta^{*}$ is constant between $t_{c}$ and $t_{d}$ :

$$
\delta^{*}\left(t_{c}\right)=\delta^{*}\left(t_{d}\right)=\delta\left(t_{c}\right)=\frac{\delta_{0}}{1-\beta D\left(t_{c}\right)}
$$

Because of the sub-critical damage growth between points $d$ and $e$, it results $D(t)>$ $D\left(t_{c}\right)$ for $t_{d}<t<t_{e}$, which yields:

$$
\frac{\delta_{0}}{1-\beta D(t)}>\frac{\delta_{0}}{1-\beta D\left(t_{c}\right)}=\max _{\tau \leq t} \delta(\tau)
$$

and from Equation (4) one obtains that:

$$
\delta^{*}(t)=\frac{\delta_{0}}{1-\beta D(t)} \quad \text { and } \quad \dot{\delta}^{*}>0
$$

At point $e$, although the displacement has reached again the maximum previously attained value, it results $\delta\left(t_{e}\right)<\delta^{*}\left(t_{e}\right)$ because of the increase in $\delta^{*}$ between points $d$ and $e$. Between points $e$ and $f$ unloading occurs again and the straight line to the origin is again followed in the traction-relative displacement plot, but with a reduced slope with respect to the first unloading because of the (sub-critical) increase in damage.

Between points $f$ and $g$ re-loading occurs again. For $t_{f}<t<t_{g}$ it results

$$
\max _{\tau \leq t} \delta(\tau)<\frac{\delta_{0}}{1-\beta D(t)} \quad \Rightarrow \quad \delta^{*}(t)=\frac{\delta_{0}}{1-\beta D(t)}
$$

Instead, the equality between the two terms is reached at point $g$, after which the damage growth is again monotonic, resulting in linear softening, until cohesion is completely lost at point $h$ and $D=0$, followed by zero traction. 


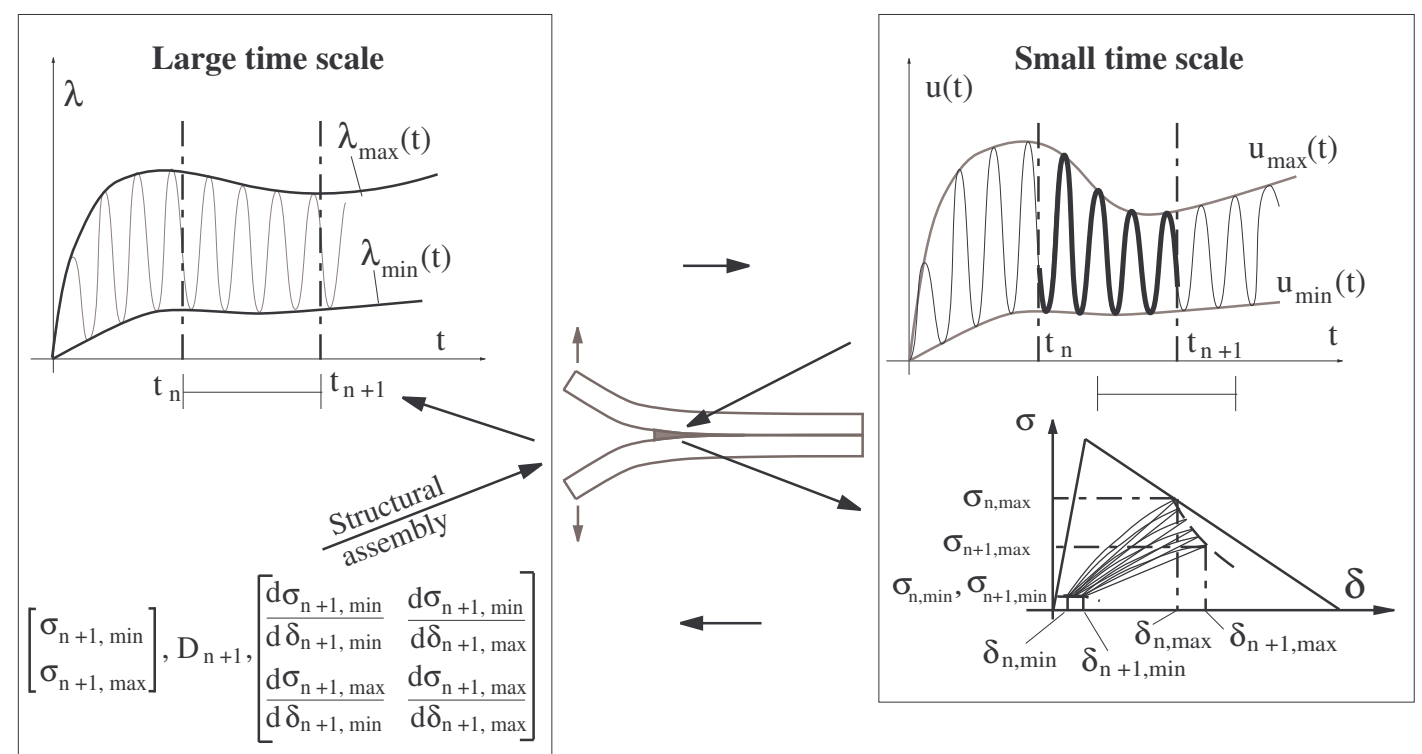

Figure 4: Schematic of the multi-time-scale solution strategy.

\section{MULTI-TIME-SCALE SOLUTION STRATEGY}

In this section the main original contribution of this work, i.e. the multi-time-scale solution scheme schematised in Figure 4, is described in detail.

It is assumed that loading process is one-dimensional, i.e. the applied external forces and the prescribed displacements are proportional to one scalar load multiplier $\lambda$, function of time, as is typical of most engineering applications. Furthermore, loading is assumed to be cyclic with constant frequency $f$ but not necessarily with constant amplitude or mean value. In particular, the following variation of $\lambda$ with time is assumed:

$$
\lambda(t)=\lambda_{m}(t)+\Delta \lambda(t) \sin (2 \pi f t)
$$

where $\lambda_{m}(t)$ and $\Delta \lambda(t)$ will be denoted as the 'instantaneous' mean value and semiamplitude, respectively, which in general can vary with time. In addition, it is assumed that $\Delta \lambda(t)>0$.

Minimum and maximum 'envelope' functions of the load multiplier, $\lambda_{\min }$ and $\lambda_{\max }$, are introduced as follows:

$$
\lambda_{\text {min }}(t)=\lambda_{m}(t)-\Delta \lambda(t) \quad \lambda_{\max }(t)=\lambda_{m}(t)+\Delta \lambda(t)
$$

The total displacements $\mathbf{v}$ can be decomposed into the sum of the prescribed ones $\mathbf{u}_{p}$ and the non-prescribed ones $\mathbf{u}$, as usual:

$$
\mathbf{v}=\mathbf{u}+\mathbf{u}_{p}
$$

The assumption that the variable displacements $\mathbf{u}$ have the same type of variation as the load multiplier is made, both in the structure and at any damaging interface:

$$
\mathbf{u}(t)=\mathbf{u}_{m}(t)+\Delta \mathbf{u}(t) \sin (2 \pi f t)
$$


$\mathbf{u}_{m}(t)$ and $\Delta \mathbf{u}(t)$ being defined as the their 'instantaneous' mean value and 'semiamplitude'. The 'minimum' and 'maximum' displacements are defined as follows:

$$
\mathbf{u}_{\min }(t)=\mathbf{u}_{m}(t)-\Delta \mathbf{u}(t) \quad \mathbf{u}_{\max }(t)=\mathbf{u}_{m}(t)+\Delta \mathbf{u}(t)
$$

and, vice versa:

$$
\mathbf{u}_{m}(t)=\frac{\mathbf{u}_{\max }(t)+\mathbf{u}_{\min }(t)}{2} \quad \Delta \mathbf{u}(t)=\frac{\mathbf{u}_{\max }(t)-\mathbf{u}_{\min }(t)}{2}
$$

Remark 3.1 Assumption (14) can be seen as the combination of two hypotheses. One is that the 'shape' of the response is the same as that of the input, which is only an approximation whose validity needs to be assessed. A second hypothesis is that input and output are in phase, which is reasonable because in this work neither damping nor inertia terms are included.

Remark 3.2 It is also worth noting that $\lambda_{\min }$ and $\lambda_{\max }$ are actual envelopes of the load multiplier (but clearly not the only ones), because $\Delta \lambda>0$, whereby $\lambda_{\min }(t) \leq$ $\lambda(t) \leq \lambda_{\max }(t)$ for any $t$ and $\lambda_{\min }(t)=\lambda(t)$ and $\lambda_{\max }(t)=\lambda(t)$ at some different times $t$ as shown below in Equation (17). Instead, $\mathbf{u}_{\min }$ and $\mathbf{u}_{\max }$ could be considered envelopes only componentwise. In fact, since the sign of the components of $\Delta \mathbf{u}(t)$ is not bound to be positive, the components of $\mathbf{u}_{\min }$ and $\mathbf{u}_{\max }$ are envelopes but not necessarily minimum or maximum. Therefore, relationships (15) are a definition of $\mathbf{u}_{\min }$ and $\mathbf{u}_{\max }$, rather than a result, and subscripts 'min' and 'max' are used because $\mathbf{u}_{\min }$ and $\mathbf{u}_{\max }$ are equal to $\mathbf{u}$ at the same times when $\lambda_{\min }$ and $\lambda_{\max }$ are equal to $\lambda$, respectively:

$$
\begin{aligned}
& \mathbf{u}_{\min }(t)=\mathbf{u}(t) \quad \text { and } \quad \lambda_{\min }(t)=\lambda(t) \quad \text { for } t=\frac{3}{4 f}+\frac{k}{f} \\
& \mathbf{u}_{\max }(t)=\mathbf{u}(t) \quad \text { and } \quad \lambda_{\max }(t)=\lambda(t) \quad \text { for } t=\frac{1}{4 f}+\frac{k}{f}
\end{aligned}
$$

and $k=1,2, \ldots,+\infty$. The same remark applies to $\mathbf{q}_{\text {min }}^{\text {ext }}, \mathbf{q}_{\max }^{\text {ext }}, \mathbf{u}_{p, \min }$ and $\mathbf{u}_{p, \max }$, which are introduced below. A similar remark will also apply to $\mathbf{q}_{\text {min }}^{\text {int }}$ and $\mathbf{q}_{\text {max }}^{\text {int }}$, as explained later.

The external forces $\mathbf{q}^{e x t}$ and prescribed displacements $\mathbf{u}_{p}$ are obtained in terms of their (fixed) reference values $\mathbf{q}_{0}^{\text {ext }}$ and $\mathbf{u}_{0, p}$ as:

$$
\mathbf{q}^{e x t}(t)=\lambda(t) \mathbf{q}_{0}^{e x t} \quad \mathbf{u}_{p}(t)=\lambda(t) \mathbf{u}_{0, p}
$$

The 'minimum' and 'maximum' external forces and prescribed displacements are defined by:

$$
\begin{aligned}
& \mathbf{q}_{\text {min }}^{\text {ext }}(t)=\lambda_{\min }(t) \mathbf{q}_{0}^{\text {ext }} \quad \mathbf{q}_{\max }^{\text {ext }}(t)=\lambda_{\max }(t) \mathbf{q}_{0}^{\text {ext }} \\
& \mathbf{u}_{p, \min }(t)=\lambda_{\min }(t) \mathbf{u}_{0, p} \quad \mathbf{u}_{p, \max }(t)=\lambda_{\max }(t) \mathbf{u}_{0, p}
\end{aligned}
$$

Assuming that the displacements $\mathbf{u}$ were known at each time $t$, the internal forces $\mathbf{q}^{\text {int }}(t)$ can be computed and two 'minimum' and 'maximum' internal force vectors, 
$\mathbf{q}_{\text {min }}^{\text {int }}$ and $\mathbf{q}_{\text {max }}^{\text {int }}$ can be introduced so that they satisfy the following conditions, analogous to relationships (17):

$$
\mathbf{q}_{\text {min }}^{\text {int }}(t)=\mathbf{q}^{\text {int }}(t) \quad \text { for } t=\frac{3}{4 f}+\frac{k}{f} \quad \mathbf{q}_{\text {max }}^{\text {int }}(t)=\mathbf{q}^{\text {int }}(t) \quad \text { for } t=\frac{1}{4 f}+\frac{k}{f}
$$

and $k=1,2, \ldots,+\infty$.

In this work the structural behaviour is assumed to be elastic apart from the damaging interface. Therefore, denoting by a superscript $e$ the element vectors from now on, it is immediate to verify that for all the finite elements $e$ except for the interface elements the element internal forces $\mathbf{q}^{\text {int,e }}$ have the same type of variation as the displacements,

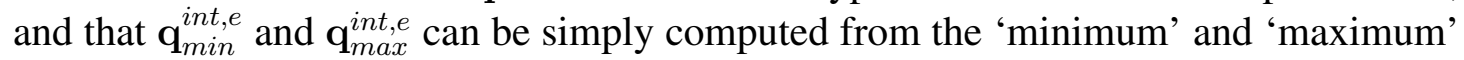
element displacements in the usual way:

$$
\begin{aligned}
& \mathbf{q}_{\text {min }}^{\text {int,e }}(t)=\left[\int_{\Omega^{e}}\left(\mathbf{B}^{e}\right)^{T} \mathbf{D} \mathbf{B}^{e} d \Omega\right]\left(\mathbf{u}_{\min }(t)+\lambda_{\min }(t) \mathbf{u}_{0, p}^{e}\right) \\
& \mathbf{q}_{\text {max }}^{\text {int,e }}(t)=\left[\int_{\Omega^{e}}\left(\mathbf{B}^{e}\right)^{T} \mathbf{D} \mathbf{B}^{e} d \Omega\right]\left(\mathbf{u}_{\max }(t)+\lambda_{\max }(t) \mathbf{u}_{0, p}^{e}\right)
\end{aligned}
$$

$\mathbf{D}$ and $\mathbf{B}^{e}$ representing the standard elastic-moduli matrix and the usual matrix relating the element nodal displacements to the element strains, respectively, while $\Omega^{e}$ denotes the element domain.

Instead, for the interface elements, the element internal forces have not necessarily the same type of variation as the displacements, and $\mathbf{q}_{\text {min }}^{\text {int,e }}$ and $\mathbf{q}_{\text {max }}^{\text {int,e }}$ must generally be determined by computing $\mathbf{q}^{\text {int,e }}(t)$, or determining an approximation of it, through the CZM of Section 2 and using relationships (20) at the element level. In this case, $\mathbf{q}_{\text {min }}^{\text {int,e }}$

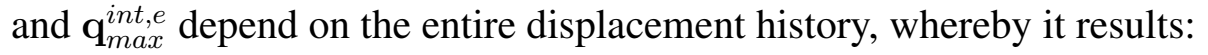

$$
\begin{aligned}
& \mathbf{q}_{\text {min }}^{\text {int,e }}=\mathbf{q}_{\text {min }}^{\text {int,e }}\left(\mathbf{u}_{\min }, \mathbf{u}_{\max }, \lambda_{\min }, \lambda_{\max }\right) \\
& \mathbf{q}_{\text {max }}^{\text {int,e }}=\mathbf{q}_{\text {max }}^{\text {int,e }}\left(\mathbf{u}_{\min }, \mathbf{u}_{\max }, \lambda_{\min }, \lambda_{\max }\right)
\end{aligned}
$$

In general, not only does Remark 3.2 apply to $\mathbf{q}_{\text {min }}^{\text {int }}$ and $\mathbf{q}_{\text {max }}^{\text {int }}$, too, but the components of $\mathbf{q}_{\text {min }}^{\text {int }}$ and $\mathbf{q}_{\text {max }}^{\text {int }}$ may not necessarily be envelopes of $\mathbf{q}^{\text {int }}(t)$.

\subsection{Large-time-scale problem}

The large-time-scale problem consists of finding functions $\mathbf{u}_{\min }$ and $\mathbf{u}_{\max }$ so that the following equilibrium equations are satisfied:

$$
\left\{\begin{array}{l}
\mathbf{r}_{\min }=\mathbf{q}_{\min }^{\text {ext }}-\mathbf{q}_{\min }^{\text {int }}=0 \\
\mathbf{r}_{\max }=\mathbf{q}_{\max }^{\text {ext }}-\mathbf{q}_{\max }^{\text {int }}=0
\end{array}\right.
$$

$\mathbf{r}_{\min }$ and $\mathbf{r}_{\max }$ denoting the 'min' and 'max' parts of the residual. The two problems are coupled at the interface because of the dependence of each of $\mathbf{q}_{\text {min }}^{\text {int,e }}$ and $\mathbf{q}_{\text {max }}^{\text {int,e }}$ in the interface elements on both $\mathbf{u}_{\min }$ and $\mathbf{u}_{\max }$ in Equation (22). 
Using the Newton-Raphson method, let us denote by $t_{n}$ and $t_{n+1}$ the times at the beginning and at the end of increment $n$ of the analysis. It is worth noting that each of these times $t_{n}$ and $t_{n+1}$ can be taken according with the chosen time-incrementation scheme, without additional constraints (i.e. they do not have to correspond to the maximum, minimum or any other specific point within a cycle). For each element $e$, the initial interface-element nodal displacements $\mathbf{u}_{n, \max }^{e}$ and $\mathbf{u}_{n, \min }^{e}$ are known and tentative values $\mathbf{u}_{n+1, \max }^{e}$ and $\mathbf{u}_{n+1, \text { min }}^{e}$ at the end of the increment have been computed after the previous iteration. For the elastic elements around the interface the internal forces can be computed in a straightforward way via Equations (21). This is not the case for the interface elements, where at each integration point of each element $e$ the maximum and minimum relative displacements at times $t_{n}$ and $t_{n+1}$ are given through the usual matrix $\mathbf{B}^{e}$, evaluated at the integration point:

$$
\begin{array}{rlrl}
\delta_{n, \max } & =\mathbf{B}^{e} \mathbf{u}_{n, \max }^{e} & \delta_{n+1, \max }=\mathbf{B}^{e} \mathbf{u}_{n+1, \max }^{e} \\
\delta_{n, \min }=\mathbf{B}^{e} \mathbf{u}_{n, \min }^{e} & \delta_{n+1, \min }=\mathbf{B}^{e} \mathbf{u}_{n+1, \min }^{e}
\end{array}
$$

In order to determine the element internal forces $\mathbf{q}_{n+1, \min }^{\text {int,e }}=\mathbf{q}_{\text {min }}^{\text {int,e }}\left(t_{n+1}\right)$ and $\mathbf{q}_{n+1, \max }^{\text {int,e }}=$ $\mathbf{q}_{\text {max }}^{\text {int, }}\left(t_{n+1}\right)$ the cyclic variation of the relative displacement is considered and the resulting variation of the interface stress and therefore of the element internal forces $\mathbf{q}^{\text {int,e }}$ is calculated as described in the next section.

Although not essential for the accurate solution of the structural problem, if the quadratic convergence of the Newton-Raphson method is to be preserved the derivatives of the residual vectors with respect to $\mathbf{u}_{n+1, \min }^{e}$ and $\mathbf{u}_{n+1, \max }^{e}$ are needed, so that the linear system to solve at each iteration is:

$$
\left[\begin{array}{ll}
\left(\frac{\partial \mathbf{q}_{n+1, \min }^{\text {int }}}{\partial \mathbf{u}_{n+1, \min }}\right)^{(k)} & \left(\frac{\partial \mathbf{q}_{n+1, \min }^{\text {int }}}{\partial \mathbf{u}_{n+1, \max }}\right)^{(k)} \\
\left(\frac{\partial \mathbf{q}_{n+1, \max }^{\text {int }}}{\partial \mathbf{u}_{n+1, \min }}\right)^{(k)} & \left(\frac{\partial \mathbf{q}_{n+1, \max }^{\text {int }}}{\partial \mathbf{u}_{n+1, \max }}\right)^{(k)}
\end{array}\right]\left[\begin{array}{l}
\mathbf{u}_{n+1, \min }^{(k+1)}-\mathbf{u}_{n+1, \min }^{(k)} \\
\mathbf{u}_{n+1, \max }^{(k+1)}-\mathbf{u}_{n+1, \max }^{(k)}
\end{array}\right]=\left[\begin{array}{c}
-\mathbf{r}_{\min }^{(k)} \\
-\mathbf{r}_{\max }^{(k)}
\end{array}\right]
$$

where superscripts $(k)$ and $(k+1)$ indicate that quantities are evaluated at iterations $(k)$ or $(k+1)$. The coefficient matrix of this linear system is the 'expanded' tangent stiffness matrix. In fact, until the response of the interface is elastic, the off-diagonal blocks of the tangent matrix are zero, and the 'min' and 'max' problems are uncoupled. Instead, when damage develops on the interface, the related interface elements will generally contribute with non-zero coupling terms in the off-diagonal blocks.

\subsection{Cycle-by-cycle time integration at the small time scale}

The variation during the increment of the 'instantaneous' mean value and semi-amplitude of the relative displacement, $\delta_{m}$ and $\Delta \delta_{m}$, are obtained by first computing these values at the beginning and at the end of the increment

$$
\begin{array}{ll}
\delta_{m}\left(t_{n}\right)=\frac{\delta_{n, \max }+\delta_{n, \min }}{2} & \Delta \delta_{m}\left(t_{n}\right)=\frac{\delta_{n, \max }-\delta_{n, \min }}{2} \\
\delta_{m}\left(t_{n+1}\right)=\frac{\delta_{n+1, \max }+\delta_{n+1, \min }}{2} & \Delta \delta_{m}\left(t_{n+1}\right)=\frac{\delta_{n+1, \max }-\delta_{n+1, \min }}{2}
\end{array}
$$


and then by linear interpolation:

$$
\begin{array}{ll}
\delta_{m}(t)=\left(\frac{t_{n+1}-t}{t_{n+1}-t_{n}}\right) \delta_{m}\left(t_{n}\right)+\left(\frac{t-t_{n}}{t_{n+1}-t_{n}}\right) \delta_{m}\left(t_{n+1}\right) & \\
\Delta \delta_{m}(t)=\left(\frac{t_{n+1}-t}{t_{n+1}-t_{n}}\right) \Delta \delta_{m}\left(t_{n}\right)+\left(\frac{t-t_{n}}{t_{n+1}-t_{n}}\right) \Delta \delta_{m}\left(t_{n+1}\right) &
\end{array} \quad t \in\left(t_{n}, t_{n+1}\right)
$$

Using the CZM, one needs to determine the stress variation $\sigma(t)$ within the increment resulting from the following assigned relative-displacement variation:

$$
\delta(t)=\delta_{m}(t)+\Delta \delta_{m}(t) \sin (2 \pi f t) \quad t \in\left(t_{n}, t_{n+1}\right)
$$

and the known initial value of the damage $D\left(t_{n}\right)$.

Pre-multimplication of $\sigma(t)$ by $\left(\mathbf{B}^{e}\right)^{T}$ and integration provide the internal element forces $\mathbf{q}^{i n t, e}$, from which $\mathbf{q}_{\text {min }}^{\text {int,e }}$ and $\mathbf{q} \mathbf{q}_{\text {max }}^{\text {int,e }}$ can be computed using relationships (20). An equivalent but simpler to implement procedure is to determine minimum and maximum interface stresses $\sigma_{\min }(t)$ and $\sigma_{\max }(t)$ that fulfill the following relationships analogous to $(20)$ :

$$
\sigma_{\min }(t)=\sigma(t) \quad \text { for } t=\frac{3}{4 f}+\frac{k}{f} \quad \sigma_{\max }(t)=\sigma(t) \quad \text { for } t=\frac{1}{4 f}+\frac{k}{f}
$$

Premultimplication of $\sigma_{\min }(t)$ and $\sigma_{\max }(t)$ by $\left(\mathbf{B}^{e}\right)^{T}$ and integration directly provide $\mathbf{q}_{\text {min }}^{\text {int, }}$ and $\mathbf{q}_{\text {max }}^{\text {int, }}$.

In this way, the element contribution to the element tangent stiffness matrix of system (25) is given by:

$$
\begin{aligned}
& {\left[\begin{array}{ll}
\left(\frac{\partial \mathbf{q}_{n+1, \text { min }}^{\text {int,e }}}{\partial \mathbf{u}_{n+1, \text { min }}}\right)^{(k)} & \left(\frac{\partial \mathbf{q}_{n+1, \text { min }}^{\text {int,e }}}{\partial \mathbf{u}_{n+1, \max }}\right)^{(k)} \\
\left(\frac{\partial \mathbf{q}_{n+1, \max }^{\text {int, }}}{\partial \mathbf{u}_{n+1, \min }}\right)^{(k)} & \left(\frac{\partial \mathbf{q}_{n+1, \max }^{\text {int,e }}}{\partial \mathbf{u}_{n+1, \max }}\right)^{(k)}
\end{array}\right]=} \\
& =\int_{\Omega_{e}}\left[\begin{array}{cc}
\left(\mathbf{B}^{e}\right)^{T}\left(\frac{\partial \sigma_{n+1, \text { min }}}{\partial \delta_{n+1, \text { min }}}\right)^{(k)} \mathbf{B}^{e} & \left(\mathbf{B}^{e}\right)^{T}\left(\frac{\partial \sigma_{n+1, \text { min }}}{\partial \delta_{n+1, \text { max }}}\right)^{(k)} \mathbf{B}^{e} \\
\left(\mathbf{B}^{e}\right)^{T}\left(\frac{\partial \sigma_{n+1, \text { max }}}{\partial \delta_{n+1, \text { min }}}\right)^{(k)} \mathbf{B}^{e} & \left(\mathbf{B}^{e}\right)^{T}\left(\frac{\partial \sigma_{n+1, \text { max }}}{\partial \delta_{n+1, \text { max }}}\right)^{(k)} \mathbf{B}^{e}
\end{array}\right] d \Omega
\end{aligned}
$$

where $\sigma_{n+1, \min }=\sigma_{\min }\left(t_{n+1}\right)$ and $\sigma_{n+1, \max }=\sigma_{\max }\left(t_{n+1}\right)$.

Remark 3.3 The time-integration of the CZM can be carried out in many different ways and, possibly, introducing some additional approximation. In fact, although the hysteretic CZM was presented in Section 2 before describing the multi-time-scale solution scheme for the sake of clarity, the proposed procedure can be employed even if a different CZM is used, such as one of those proposed in Refs. $[6-9,11]$. Hence, the 
detailed solution of the CZM to determine $\sigma_{n+1, \min }$ and $\sigma_{n+1, \max }$ at each integration point is separately reported in the next section, because it is one of the many possible choices. Exploration of other methods and the investigation on the implications of different time-integration schemes or indeed other CZMs is left for future work. Furthermore, the range of applicability of the proposed multi-time-scale strategy is clearly much wider than that addressed in this paper, and includes all problems involving fatigue damage and failure.

\section{CLOSED-FORM APPROXIMATE TIME-INTEGRATION OF THE PROPOSED CZM}

This section presents a closed-form solution scheme for the small-time-scale problem described in Section 3.2 for the case when the CZM of Section 2 is used. The relative displacements $\delta_{n, \min }, \delta_{n, \max }, \delta_{n+1, \min }$ and $\delta_{n+1, \max }$ and the damage $D_{n}=D\left(t_{n}\right)$ are assigned. The following developments will be made assuming that both $\delta_{n, \min }$ and $\delta_{n+1, \min }$ are non negative, both for the sake of simplicity and because this was always the case in the numerical applications considered for those integration points where $D_{n}>0$.

Let us first consider the case that $\delta_{n+1, \max } \leq \delta_{n, \max }$. This is schematised in Figure 5, where, instead, the further assumption that $\delta_{n+1, \min } \geq \delta_{n, \min }$ is immaterial because what follows also applies when $\delta_{n+1, \min }<\delta_{n, \min }$. In this case, regardless of whether or not the previous damage increase has been monotonic, that is of whether or not $\delta^{*}\left(t_{n}\right)=$ $\delta_{n, \max }$, the linear interpolation (27) implies that the maximum relative displacement never increases beyond $\delta^{*}\left(t_{n}\right)$. Hence, there will be no monotonic damage growth and all the damage increase will be sub-critical.

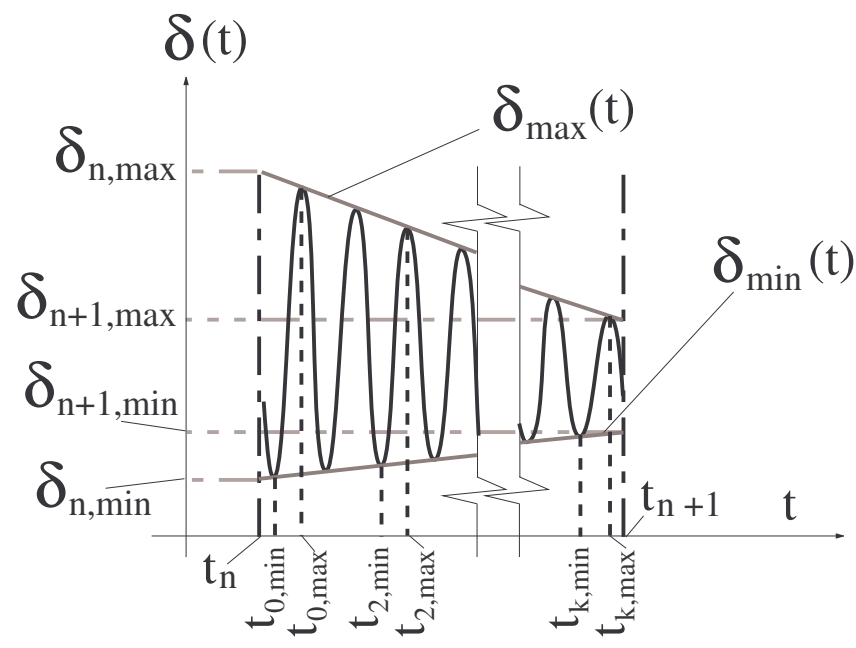

Figure 5: cyclic relative-displacement variation entailing no monotonic damage growth.

Let us assume that the number of local minima and maxima of $\delta$ in the increment 
$\left(t_{n}, t_{n+1}\right)$ are the same and equal to $k+1$. Furthermore, let $t_{0, \min }, t_{0, \max }, t_{1, \min }, t_{1, \max }$, $\ldots, t_{k, \min }, t_{k, \max }$ be the times when the local minima and maxima are attained, respectively, assuming that $t_{j, \min }<t_{j, \max }$ as shown in Figure 5. Equation (5) is easily integrated between each local maximum and the next local maximum by observing that between $t_{j-1, \max }$ and $t_{j, \min }$ Equation (5) $)_{4}$ applies and no damage growth occurs, while between $t_{j, \min }$ and $t_{j, \max }$ Equation (5) $)_{5}$ is used. This results in the following recursive expression:

$$
\begin{gathered}
D\left(t_{1, \text { max }}\right)=D\left(t_{0, \text { max }}\right) e^{\gamma\left[\delta\left(t_{1, \text { max }}\right)-\delta\left(t_{1, \text { min }}\right)\right]} \\
D\left(t_{2, \text { max }}\right)=D\left(t_{1, \text { max }}\right) e^{\gamma\left[\delta\left(t_{2, \text { max }}\right)-\delta\left(t_{2, \text { min }}\right)\right]} \\
\cdots \\
D\left(t_{k, \text { max }}\right)=D\left(t_{k-1, \max }\right) e^{\gamma\left[\delta\left(t_{k, \text { max }}\right)-\delta\left(t_{k, \text { min }}\right)\right]}
\end{gathered}
$$

which yields:

$$
D\left(t_{k, \max }\right)=D\left(t_{0, \max }\right) e^{\gamma \sum_{j=1}^{k}\left[\delta\left(t_{j, \max }\right)-\delta\left(t_{j, \min }\right)\right]}
$$

By dividing and multiplying the sum by $k$, and observing that $\delta\left(t_{j, \max }\right)=\delta_{\max }\left(t_{j, \max }\right)$ and $\delta\left(t_{j, \min }\right)=\delta_{\min }\left(t_{j, \min }\right)$, one obtains:

$$
\sum_{j=1}^{k}\left[\delta\left(t_{j, \max }\right)-\delta\left(t_{j, \min }\right)\right]=\left(\frac{\sum_{j=1}^{k} \delta_{\max }\left(t_{j, \max }\right)}{k}-\frac{\sum_{j=1}^{k} \delta_{\min }\left(t_{j, \min }\right)}{k}\right) k
$$

The two sums in the last term are the averages of $\delta_{\max }\left(t_{j, \max }\right)$ and $\delta_{\min }\left(t_{j, \min }\right)$, for $j$ ranging from 1 to $k$. For $k$ sufficiently big we can approximate:

$$
\begin{aligned}
& \frac{\sum_{j=1}^{k} \delta_{\text {max }}\left(t_{j, \text { max }}\right)}{k}=\frac{\sum_{j=0}^{k} \delta_{\text {max }}\left(t_{j, \text { max }}\right)}{k+1} \\
& \frac{\sum_{j=1}^{k} \delta_{\text {min }}\left(t_{j, \text { min }}\right)}{k}=\frac{\sum_{j=0}^{k} \delta_{\text {min }}\left(t_{j, \text { min }}\right)}{k+1}
\end{aligned}
$$

Because of the linear interpolation (27) $\delta_{\max }$ and $\delta_{\min }$ are linear within the increment, so that it results:

$$
\begin{aligned}
& \frac{\sum_{j=0}^{k} \delta_{\max }\left(t_{j, \max }\right)}{k+1}=\frac{\delta_{\max }\left(t_{0, \max }\right)+\delta_{\max }\left(t_{k, \max }\right)}{2} \\
& \frac{\sum_{j=0}^{k} \delta_{\min }\left(t_{j, \min }\right)}{k+1}=\frac{\delta_{\min }\left(t_{0, \min }\right)+\delta_{\min }\left(t_{k, \min }\right)}{2}
\end{aligned}
$$

Since $k=f\left(t_{k, \max }-t_{0, \max }\right), f$ being the frequency of the cyclic loading, Equation (32) can be rewritten as follows:

$D\left(t_{k, \max }\right)=D\left(t_{0, \max }\right) e^{0.5 \gamma f\left(t_{k, \max }-t_{0, \max }\right)\left[\delta_{\max }\left(t_{0, \max }\right)+\delta_{\max }\left(t_{k, \max }\right)-\delta_{\min }\left(t_{0, \min }\right)-\delta_{\min }\left(t_{k, \min }\right)\right]}$ 
Assuming again that $k$ is sufficiently big, or that the variations of $\delta_{\min }$ within half a cycle is negligible, we can make the following approximation:

$$
\delta_{\min }\left(t_{0, \min }\right)=\delta_{\min }\left(t_{0, \max }\right) \quad \delta_{\min }\left(t_{k, \min }\right)=\delta_{\min }\left(t_{k, \max }\right)
$$

which yields:

$D\left(t_{k, \max }\right)=D\left(t_{0, \max }\right) e^{0.5 \gamma f\left(t_{k, \max }-t_{0, \max }\right)\left[\delta_{\max }\left(t_{0, \max }\right)+\delta_{\max }\left(t_{k, \max }\right)-\delta_{\min }\left(t_{0, \max }\right)-\delta_{\min }\left(t_{k, \max }\right)\right]}$

This last formula provides the sub-critical damage growth between the first and the last local maxima within the increment, in closed form. Since the formula can also be written replacing $t_{0, \max }$ and $t_{k, \max }$ with two times which do not generally coincide with local maxima, it seems reasonable to do so replacing these times with $t_{n}$ and $t_{n+1}$, i.e. to extrapolate the result over the entire time increment. This provides the value of the damage at the end of the increment as follows:

$$
D_{n+1}=D\left(t_{n+1}\right)=D\left(t_{n}\right) e^{0.5 \gamma f\left(t_{n+1}-t_{n}\right)\left[\delta_{n, \max }+\delta_{n+1, \max }-\delta_{n, \min }-\delta_{n+1, \min }\right]}
$$

At the end of the increment $\delta^{*}\left(t_{n+1}\right)$ has changed because of the sub-critical damage growth, in accordance with relationship (4), and it results:

$$
\delta^{*}\left(t_{n+1}\right)=\frac{\delta_{0}}{1-\beta D\left(t_{n+1}\right)}
$$

Remark 4.1 From the above derivation it turns out that the bigger $k$, that is the bigger the number of cycles within one increment, the better the approximation achieved. This might suggest that a link exists between the solution procedure proposed in this paper and the use of asymptotic expansion techniques, such as the multi-scale approach proposed by Fish and Oskay [29]. On the other hand, the procedures differ for many other aspects and drawing a parallel is beyond the scope of this work. More generally, in consideration of the novelty of the proposed method and of the very promising numerical results presented in Section 5 and obtained with this first proposed implementation, further studies to provide a more rigorous theoretical background to the solution procedure proposed in this paper are also left for future work, which will include error estimations related to assumption (14) and the exploration of alternative solution schemes for the small-time-scale solution scheme at the integration-point level.

Remark 4.2 Although the formulation has been developed in the case that both $\delta_{n, \min }$ and $\delta_{n+1, \min }$ are non negative. it is easy to recognise that the case when both $\delta_{n, \min }$ and $\delta_{n+1, \min }$ are non positive is of equally simple solution while the case when one is positive and the other is negative is still conceptually simple to handle but results in a more complex final expression.

In the case that $\delta_{n+1, \max }>\delta_{n, \max }$, it is not known a priori if the damage increase is monotonic or sub-critical within the increment. Hence, the assumption that the damage increase is sub-critical is made first, and a tentative value for the damage at the end of the increment is computed using Equation (39). Then, the value of $\delta^{*}\left(t_{n+1}\right)$ given by Equation (40) is compared with $\delta_{n+1, \max }$. If $\delta_{n+1, \max }<\delta^{*}\left(t_{n+1}\right)$, then the damage 
increase is indeed sub-critical and the computed value of the damage is the correct one. Instead, if $\delta_{n+1, \max }>\delta^{*}\left(t_{n+1}\right)$ the damage increase is monotonic and $(2)_{2}$ yields:

$$
D_{n+1}=\frac{\delta_{n+1, \max }-\delta_{0}}{\beta \delta_{n+1, \max }}
$$

In any case, regardless of whether the damage increase is sub-critical or monotonic, $\sigma_{n+1, \max }$ and $\sigma_{n+1, \min }$ are finally given by:

$$
\sigma_{n+1, \max }=\left(1-D_{n+1}\right) K \delta_{n+1, \max } \quad \sigma_{n+1, \min }=\left(1-D_{n+1}\right) K \delta_{n+1, \min }
$$

\subsection{Material tangent stiffness}

The four terms of the material tangent stiffness matrix required in Equation (30) are computed by differentiating Equations (42) with respect to $\delta_{n+1, \max }$ and $\delta_{n+1, \min }$, which yields

$$
\begin{aligned}
& \frac{\partial \sigma_{n+1, \max }}{\partial \delta_{n+1, \max }}=\left[1-D_{n+1}\right] K-\frac{\partial D_{n+1}}{\partial \delta_{n+1, \max }} K \delta_{n+1, \max } \\
& \frac{\partial \sigma_{n+1, \max }}{\partial \delta_{n+1, \min }}=-\frac{\partial D_{n+1}}{\partial \delta_{n+1, \min }} K \delta_{n+1, \max } \\
& \frac{\partial \sigma_{n+1, \min }}{\partial \delta_{n+1, \max }}=-\frac{\partial D_{n+1}}{\partial \delta_{n+1, \max }} K \delta_{n+1, \min } \\
& \frac{\partial \sigma_{n+1, \min }}{\partial \delta_{n+1, \min }}=\left[1-D_{n+1}\right] K-\frac{\partial D_{n+1}}{\partial \delta_{n+1, \min }} K \delta_{n+1, \min }
\end{aligned}
$$

When sub-critical damage has been found Equation (39) yields:

$$
\begin{gathered}
\frac{\partial D_{n+1}}{\partial \delta_{n+1, \max }}=0.5 \gamma f\left(t_{n+1}-t_{n}\right) D_{n+1} \\
\frac{\partial D_{n+1}}{\partial \delta_{n+1, \min }}=-0.5 \gamma f\left(t_{n+1}-t_{n}\right) D_{n+1}
\end{gathered}
$$

Otherwise, when the damage has been found to be monotonic, Equation (41) provides:

$$
\begin{aligned}
\frac{\partial D_{n+1}}{\partial \delta_{n+1, \text { max }}} & =\frac{\delta_{0}}{\beta \delta_{n+1, \text { max }}^{2}} \\
\frac{\partial D_{n+1}}{\partial \delta_{n+1, \text { min }}} & =0
\end{aligned}
$$

\section{NUMERICAL RESULTS}

The cohesive-zone model and the multi-time-scale solution strategy described in the previous sections have been implemented in an in-house general-purpose non-linear 
finite-element code developed by the author and in this section numerical results will be presented to show their effectiveness.

The double-cantilever-beam (DCB) adhesive joint studied by Shyanbhog [30] and made of two aluminium plates joined by the epoxy adhesive Araldite ${ }^{R} 2013$ is analysed. The tested specimen, which is shown in Figure 6, was subject to cyclic loading under displacement control with constant frequency of $1 \mathrm{~Hz}$ and constant amplitude and mean value, as detailed in Section 5.1. The geometry and loading scheme used are reported in Figure 7, while the material parameters used in the analysis are reported in Table 1. The interface strength $\sigma_{0}$ was estimated based on the adhesive tensile strength, while $\beta$ was chosen so as to obtain a very high initial penalty value for the interface stiffness. It was found that $\beta$ and $\sigma_{0}$ are high enough for the results to be effectively not affected by small changes of them, in accordance with the findings reported in [4]. The fracture energy $G_{c}$ was determined by curve-fitting the results of a monotonic test. Instead, $\gamma$ was calibrated by comparing experimental and numerical results.

Because of symmetry only the top half of the geometry was analysed, using a regular mesh made of two rows of 808 -noded, iso-parametric, plane-strain elements for the aluminium plate and one row of 65 6-noded, iso-parametric interface elements.

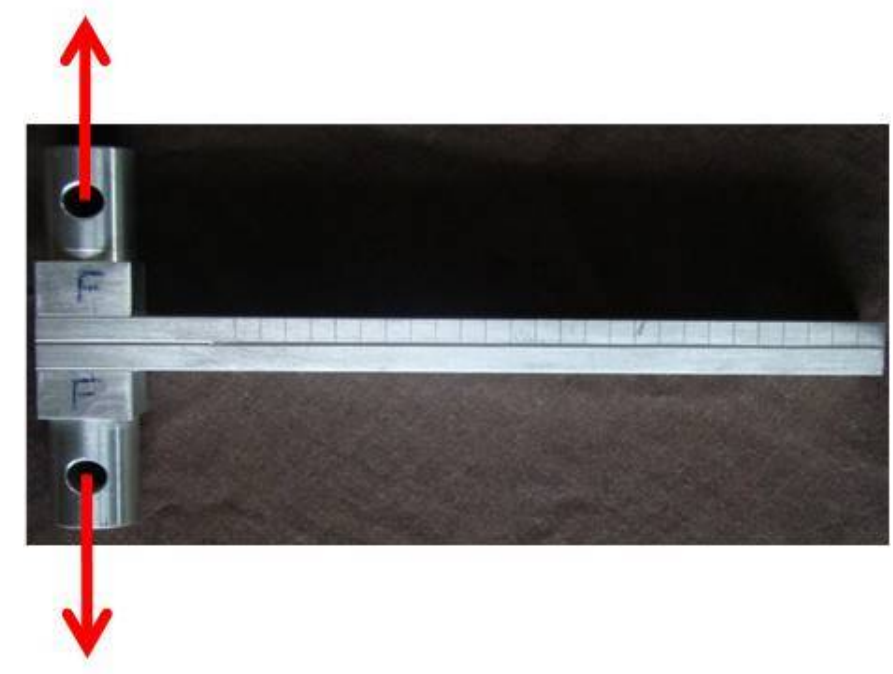

Figure 6: DCB adhesive joint tested [30].

\begin{tabular}{|c|c|c|c|c|c|}
\hline \multicolumn{2}{|c|}{ Aluminium } & \multicolumn{4}{|c|}{ Interface } \\
\hline Young modulus (GPa) & Poisson ratio & $G_{c}\left(\mathrm{~N} \mathrm{~mm}^{-1}\right)$ & $\sigma_{0}(\mathrm{MPa})$ & $\beta$ & $\gamma\left(\mathrm{mm}^{-1}\right)$ \\
\hline 70 & 0.33 & 0.4 & 12 & 0.98 & 0.09 \\
\hline
\end{tabular}

Table 1: Material parameters used in the numerical simulations. 


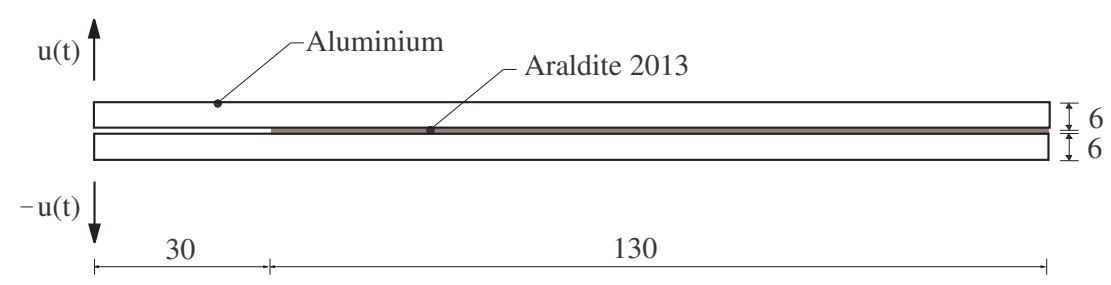

Figure 7: DCB-test geometry and loading (dimensions in $\mathrm{mm}$ ).

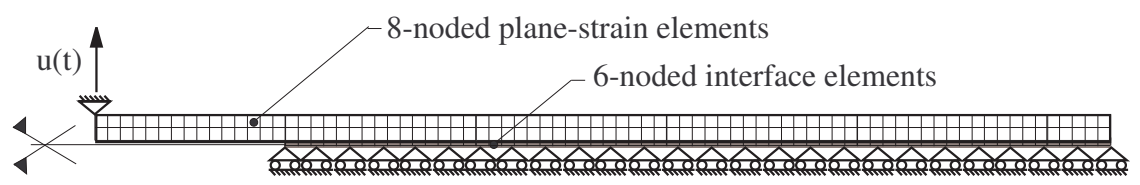

Figure 8: finite-element model used in the DCB-test.

For each of the two cases analysed and reported below in Sections 5.1 and 5.2, two types of analyses were performed, one in a conventional way and one using the multitime-scale solution procedure. In all cases the frequency of the cyclic prescribed displacements was $1 \mathrm{~Hz}$, as in the experimental test, although the rate-independence of the model make results independent from the frequency.

In the conventional analysis, the cyclic loading was divided in a number of increments, using an automatic incrementation scheme with a maximum time increment of $0.033 \mathrm{~s}$ to have about 30 increments per cycle, in order to have good sampling and to accurately capture the maximum and minimum reactions. The CZM of Section 2 was implemented in the 6-noded interface elements, and all elements have the standard number of degrees of freedom, namely 16 and 12 for the plane-strain and the interface elements, respectively.

In the multi-time-scale analysis, an automatic incrementation scheme was used too, with a maximum time increment of $15 \mathrm{~s}$, so that up to 15 cycles can be 'jumped over' within one increment of the analysis. As explained in Section 3, both in the plane-strain elements and in the interface elements the number of degrees of freedom needs to be doubled, in order to provide the 'maximum' and 'minimum' displacement fields $\mathbf{u}_{\max }$ and $\mathbf{u}_{\text {min }}$. Denoting again by $\mathbf{B}^{e}$ the standard matrix relating the nodal displacements to the strain at each point of the element, in the plane-strain elements the stiffness matrix is formally obtained by the same formula as Equation (30), simply using the following constant material stiffness matrix:

$$
\left[\begin{array}{ll}
\left(\frac{\partial \sigma_{n+1, \min }}{\partial \delta_{n+1, \min }}\right)^{(k)} & \left(\frac{\partial \sigma_{n+1, \min }}{\partial \delta_{n+1, \max }}\right)^{(k)} \\
\left(\frac{\partial \sigma_{n+1, \max }}{\partial \delta_{n+1, \min }}\right)^{(k)} & \left(\frac{\partial \sigma_{n+1, \max }}{\partial \delta_{n+1, \max }}\right)^{(k)}
\end{array}\right]=\left[\begin{array}{cc}
\mathbf{D}_{p s} & \mathbf{0} \\
\mathbf{0} & \mathbf{D}_{p s}
\end{array}\right]
$$

where $\mathbf{D}_{p s}$ denotes the matrix of the elastic moduli in the plane-strain case. 
In both types of analysis, a number of 'desired' iterations equal to $I_{d}=5$ was set, and the automatic incrementation procedure updated the time increment for a new increment $n+1$ based on the number of iterations $I_{n}$ required to get convergence within increment $n$, as follows:

$$
\Delta t_{n+1}=\Delta t_{n} \sqrt{\frac{I_{d}}{I_{n}}}
$$

$\Delta t_{n+1}$ and $\Delta t_{n}$ denoting the time increments at increments $n+1$ and $n$, respectively.

\subsection{Constant mean value and amplitude}

A first case in which the prescribed mean value and semi-amplitude are constant and equal to $u_{m}=0.85 \mathrm{~mm}$ and $\Delta u=0.69 \mathrm{~mm}$, respectively, was analysed both numerically and experimentally in Ref. [30]. Figure 9 shows the prescribed history, including a first initial ramp loading during the first $0.16 \mathrm{~s}$ of the analysis to reach the mean prescribed displacement.

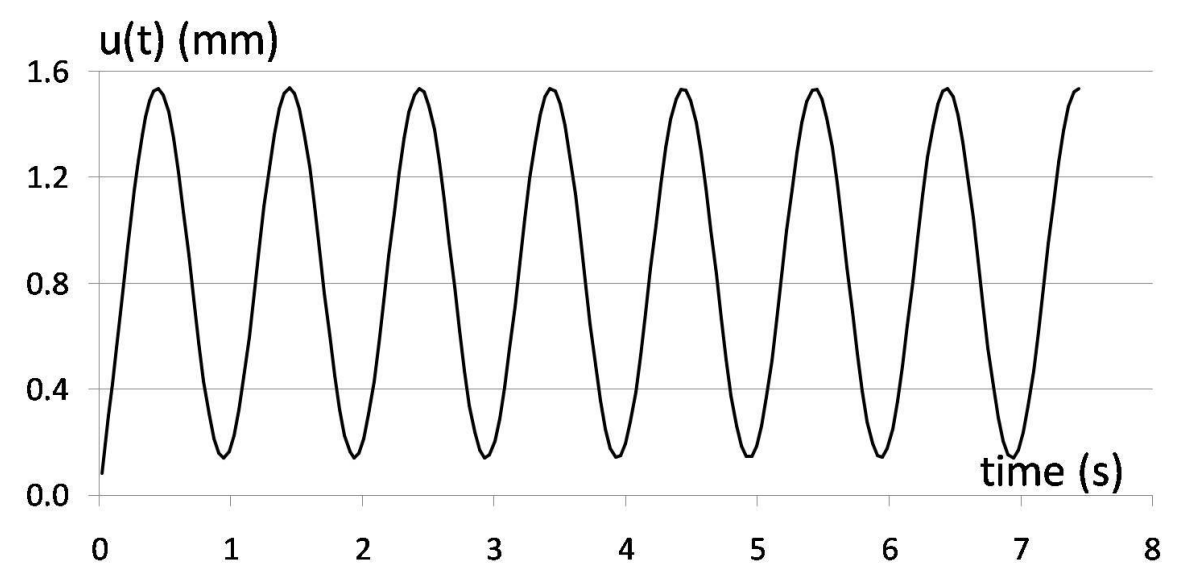

Figure 9: prescribed displacement history for the case of constant mean value and amplitude.

Figure 10 reports a comparison between the time variation of the vertical reactions obtained using the conventional cycle-by-cycle analysis and the multi-time-scale strategy. For the latter, the results are obtained in terms of two curves, which are the maximum and minimum reactions obtained for the large-scale problem as described in Section 3. Figure 10 shows excellent accuracy obtained using the multi-time-scale solution scheme.

During the first $0.16 \mathrm{~s}$ of the analysis, where the mean value and the amplitude change from zero to the final value, and during the first fourth of the first cycle, most of the damage increase is monotonic in the multi-time-scale analysis. This results in a relatively rapid increase in damage which, in turn, entails the need for a small time increment, which was set initially equal to $0.08 \mathrm{~s}$. The amplitude and the mean value remain then constant and the damage increase becomes sub-critical and therefore much slower, whereby convergence is easier and results in a gradual increase of the time increment reaching the maximum set value of $15 \mathrm{~s}$ after about 130 cycles. 
In Figure 11 a comparison between the maximum reaction obtained using the multitime-scale analysis and that measured experimentally is reported. It shows that the CZM is able to capture the behaviour reasonably well.

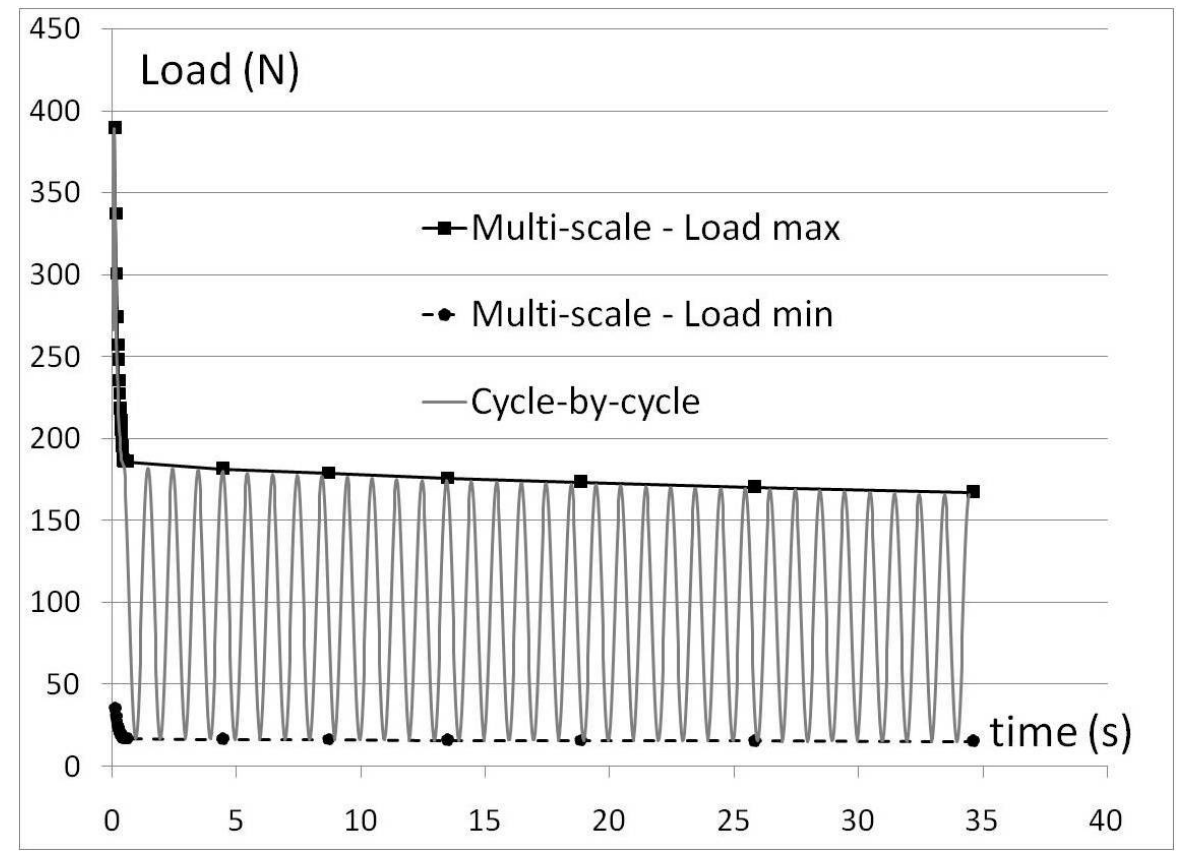

Figure 10: comparison between the reactions obtained in the vertical cycle-by-cycle and the multi-time-scale analyses for the case of constant mean value and amplitude.

Finally, the comparison between experimental and numerical analysis in terms of crack-length versus number of cycles is reported in Figure 12 and shows quite good agreement.

Considering that the main aim of this paper is not to claim that the proposed CZM is better than other similar models presented in the literature but rather to propose the original multi-time-scale solution procedure, the experimental validation is considered satisfactory. On the other hand, the proposed CZM provides some advantages in that it allows an approximated closed-form solution of the cycle-by-cycle time-integration at the small scale level, which allowed the author to focus on the overall aspects of the solution procedure leaving the further development of the CZM and of its timeintegration at the small scale for future work. Similarly, since the experimental work was limited here to the case of constant amplitude and mean value, future work will also extend the experimental testing to the case of variable amplitude and mean value, which is analysed in the next section numerically.

\subsection{Variable mean value and amplitude}

In order to further verify the numerical accuracy of the multi-time-scale solution scheme also in the case of variable mean value and amplitude, the displacement history of Figure 13 has been prescribed for the analysis. The comparison between the results obtained 


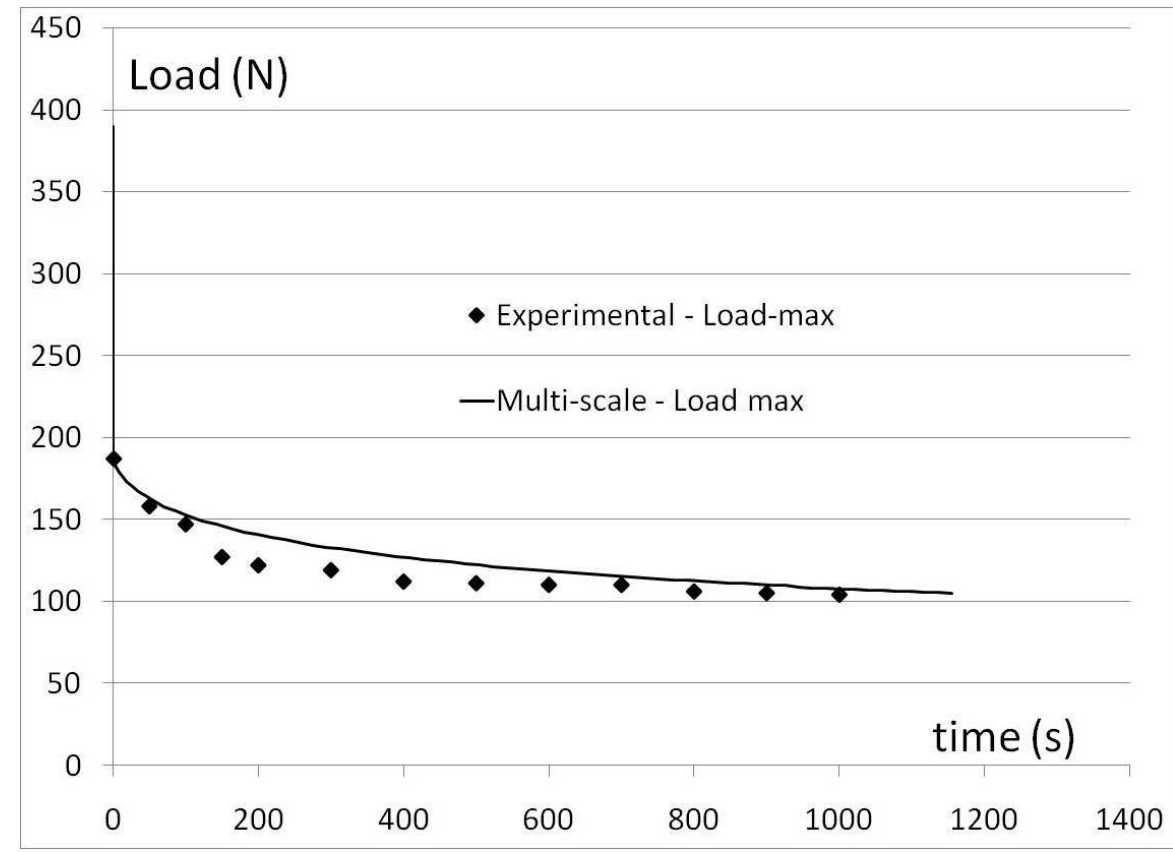

Figure 11: numerical vs experimental [30] results in terms of the time variation of the vertical reaction.

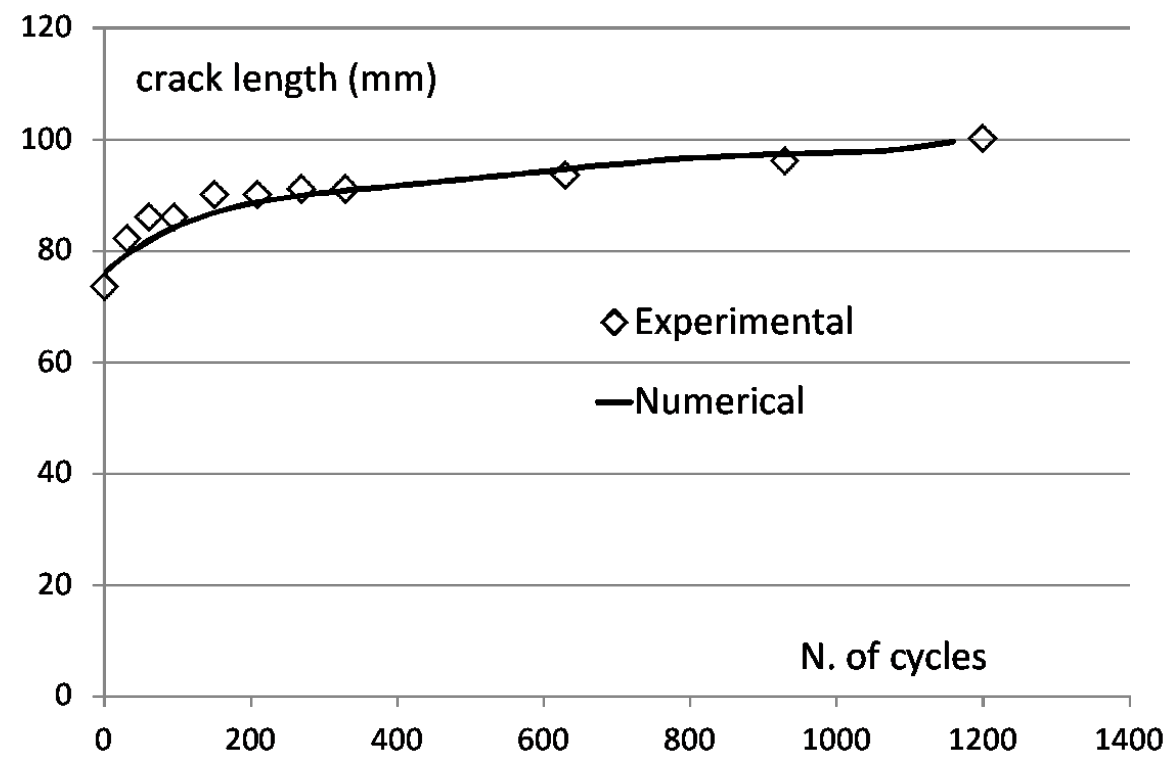

Figure 12: numerical vs experimental [30] results in terms of crack-length as a function of the number of cycles.

with the multi-time-scale and the cycle-by-cycle analyses are reported in Figure 14 and demonstrate that the accuracy obtained is still excellent.

Also in this case rapid monotonic damage growth occurs during the initial ramp load, within the first $0.16 \mathrm{~s}$, and during the first fourth of the first cycle, which entailed the use 


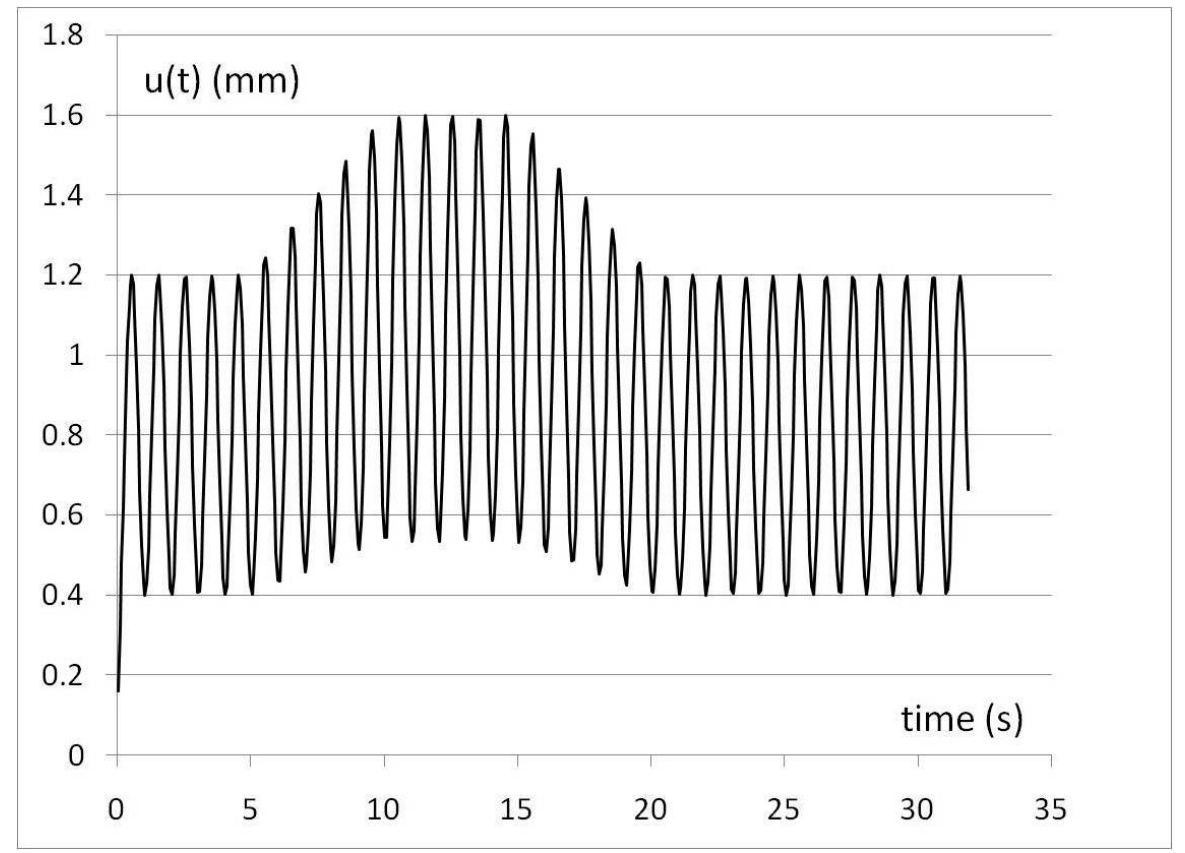

Figure 13: prescribed displacement history for the case of variable mean value and amplitude.

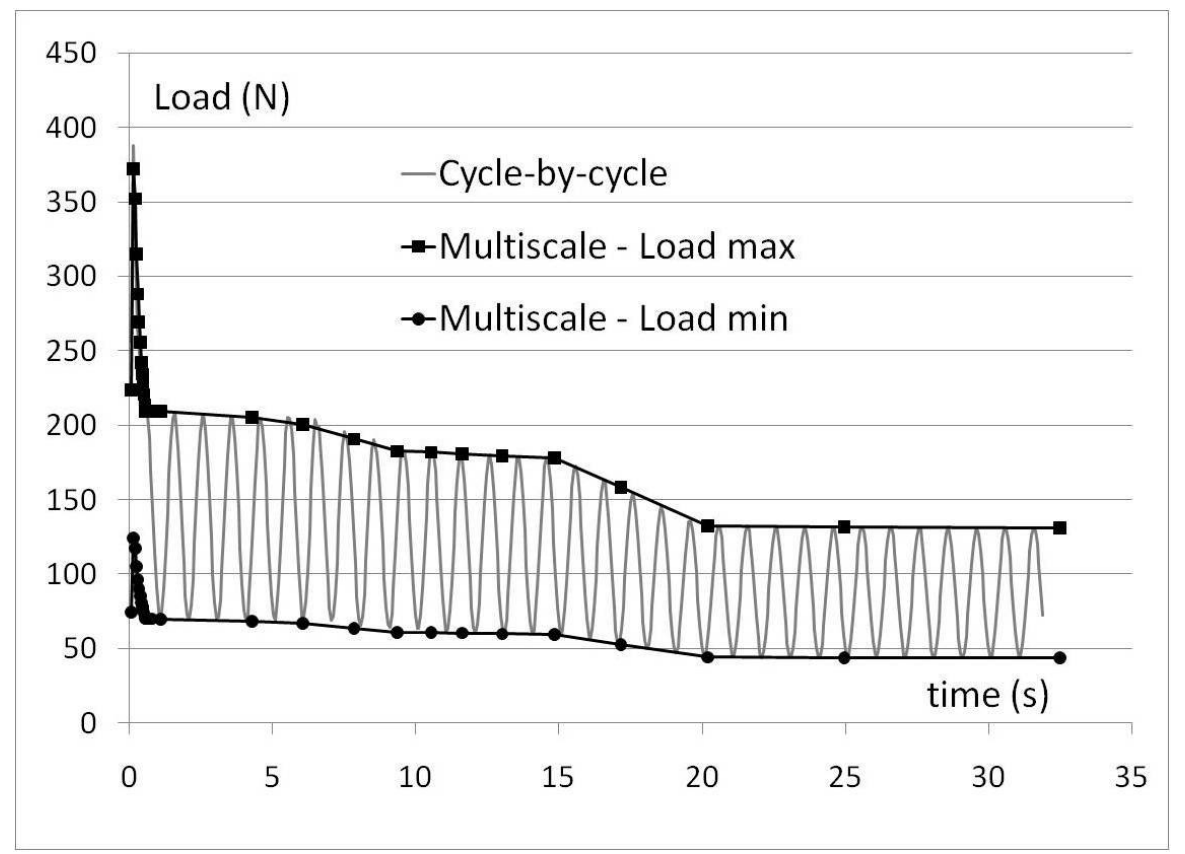

Figure 14: comparison between the vertical reactions obtained in the cycle-by-cycle and the multi-time-scale analyses for the case of variable mean value and amplitude.

of a small time increment that gradually increases between $t=0.16 \mathrm{~s}$ and $t=5 \mathrm{~s}$, when the mean value and amplitude are constant and equal to $0.8 \mathrm{~mm}$ and $0.4 \mathrm{~mm}$, respectively, 
resulting in subcritical damage growth only. Between, $t=5$ and $t=10$ the mean value and the amplitude linearly increase of a factor 1.33, reaching values of $1.067 \mathrm{~mm}$ and $0.53 \mathrm{~mm}$, so that the maximum and minimum prescribed displacements increase to $1.6 \mathrm{~mm}$ and $0.53 \mathrm{~mm}$. In this part damage growth is mostly monotonic and therefore faster, leading to a reduction of the time increment and of the load. After $t=10$, the mean value and the amplitude remain constant until $t=15$, then linearly decrease again reaching the initial value at $t=20 \mathrm{~s}$. Hence, after $t=10 \mathrm{~s}$ damage growth is sub-critical only and therefore very slow, the visible reduction in maximum and minimum reactions shown in Figure 14 between $t=15 \mathrm{~s}$ and $t=20 \mathrm{~s}$ being mainly due to the reduction in the maximum and minimum prescribed displacements. Hence, with slower damage growth, convergence is easier and the time increment increases, gradually tending to the maximum set value of $15 \mathrm{~s}$.

The most important positive aspect of the presented numerical results is the very good accuracy with which the minimum and maximum reactions are obtained using the multi-time-scale solution procedure in both of the analysed cases. As commented in Section 3, the proposed solution procedure relies on assumption (14), whose validity is not a trivial issue. Furthermore, the time-integration scheme at the small time-scale level also relies on some assumptions which are expected to be well verified when each time increment spans a large number of cycles. In fact, the numerical results show that the accuracy is excellent also when the time increment is much smaller. All these issues require further and deeper theoretical investigation to determine error estimators which can lead to a more rigorous explanation of the excellent accuracy obtained.

\section{CONCLUSIONS}

The multi-time-scale solution procedure proposed in this paper is a very effective way to analyse fatigue crack-growth using cohesive-zone models with hysteretic response and sub-critical damage evolution because it eliminates the most fundamental drawback of this type of approach, that is the excessive number of increments required in a conventional, incremental cycle-by-cycle analysis.

Despite the hypotheses made on the displacement variation in each cycle, excellent agreement is obtained between the analysis conducted with the new proposed multitime-scale method and a conventional cycle-by-cycle simulation using the same interface model. With the proposed cohesive-zone model, good agreement with experimental results in terms of load decay with time and crack length advance with time demonstrate the potential of the developed approach to become a viable and very effective tool for fatigue-life prediction in industrial applications.

The proposed multi-time-scale strategy can be applied to other cohesive-zone models, different from that used in this work and possibly including mixed-mode damage, as long as they simulate the sub-critical damage growth occurring during cyclic loading. Hence, future work will aim to get further understanding of the underlying physics of delamination or debonding due to fatigue, both to develop more accurate cohesive-zone models and to determine efficient procedures for the identification of the parameters. Furthermore deeper theoretical studies aiming to determine error estimators for the proposed solution scheme will be carried out. 
Finally, it is important to underline that the range of applicability of the proposed multi-time-scale approach is not limited to the simulation of fatigue crack growth on interfaces, but potentially comprises also other types of fatigue phenomena such as lowcycle fatigue in general and thermo-mechanical fatigue damage in metals in particular.

\section{References}

[1] P.C. Paris and F.A. Erdogan. A critical analysis of crack propagation laws. Journal of Basic Engineering - Transactions of ASME, 85:528-534, 1963.

[2] B. Harris, editor. Fatigue in Composites. Woodhead Publishing, 2003.

[3] S. Suresh. Fatigue of materials. Cambridge University Press, 1998.

[4] G. Alfano and M.A. Crisfield. Finite element interface models for the delamination analysis of laminated composites: mechanical and computational issues. International Journal for Numerical Methods in Engineering, 50(7):1701-1736, 2001.

[5] Y. Mi, M.A. Crisfield, G.A.O. Davies, and H.B. Hellweg. Progressive delamination using interface elements. Journal of Composite Materials, 32(14):1246-1272, 1998.

[6] O. Nguyen, E.A. Repetto, M. Ortiz, and R.A. Radovitzky. A cohesive model of fatigue crack growth. International Journal of Fracture, 110(4):351-369, 2001.

[7] B. Yang, S. Mall, and K. Ravi-Chandar. A cohesive zone model for fatigue crack growth in quasibrittle materials. International Journal of Solids and Structures, 38(22-23):3927-3944, 2001.

[8] K.L. Roe and T. Siegmund. An irreversible cohesive zone model for interface fatigue crack growth simulation. Engineering Fracture Mechanics, 70(2):209232, 2003.

[9] S. Maiti and P.H. Geubelle. A cohesive model for fatigue failure of polymers. Engineering Fracture Mechanics, 72(5):691-708, 2005.

[10] A. Abdul-Baqi, P.J.G. Schreurs, and Geers M.G.D. Fatigue damage modeling in solder interconnects using a cohesive zone approach. International Journal of Solids and Structures, 42(3-4):927-942, 2005.

[11] S. Serebrinsky and M. Ortiz. A hysteretic cohesive-law model of fatigue-crack nucleation. Scripta Materialia, 53(10):1193-1196, 2005.

[12] A. Jaubert and J.J. Marigo. Justification of paris-type fatigue laws from cohesive forces model via a variational approach. Continuum Mechanics and Thermodynamics, 18(1-2):23-45, 2006. 
[13] J.L. Buvard, J.L. Chaboche, F. Feyel, and F. Gallerneau. A cohesive zone model for fatigue and creep-fatigue crack growth in single crystal superalloys. International Journal of Fatigue, 31(5):868-879, 2009.

[14] R. Abdelmoula, J.J. Marigo, and Weller T. Construction of a fatigue law from a cohesive force model: the mode III case. Comptes Rendus Méchanique, 337(1):53$59,2009$.

[15] R. Abdelmoula, J.J. Marigo, and Weller T. Construction of fatigue laws from cohesive forces models: the mode I case. Comptes Rendus Méchanique, 337(3):166172, 2009.

[16] A. Ural, V.R. Krishnan, and K.D. Papoulia. A cohesive zone model for fatigue crack growth allowing for crack retardation. International Journal of Solids and Structures, 46(11-12):2453-2462, 2009.

[17] J.J. Marigo. Modeling of brittle and fatigue damage for elastic-material by gorwth of microvoids. Engineering Fracture Mechanics, 21(4):861-874, 1985.

[18] Q.D. Yang, D.J. Shim, and S.M. Spearing. A cohesive zone model for low cycle fatigue life prediction of solder joints. Microelectronic Engineering, 75(1):85-95, 2004.

[19] D. Violeau. Numerical analysis of delamination fatigue in composites via a cohesive zone model. ENS Cashan, 2001. Master's degree final report 99-05.

[20] R.H.J. Peerlings, W.A.M. Brekelmans, R. de Borst, and M.G.D. Geers. Gradientenhanced damage modelling of high-cycle fatigue. International Journal for $\mathrm{Nu}$ merical Methods in Engineering, 49(12):1547-1569, 2000.

[21] P. Robinson, U. Galvanetto, G. Bellucci, D. Tumino, and D. Violeau. Numerical simulation of fatigue-driven delamination using interface elements. International Journal for Numerical Methods in Engineering, 63(13):1824-1848, 2006.

[22] J. Muñoz, P. Robinson, and U. Galvanetto. On the numerical simulation of fatigue driven delamination with interface elements. International Journal of Fracture, 28(10):1136-1146, 2006.

[23] A. Turon, J. Costa, P.P. Camanho, and C.G. Dávila. Simulation of delamination in composites under high-cycle fatigue. Composites: part A - Applied Science and Manufacturing, 38(11):2270-2282, 2007.

[24] M. May and S.R. Hallett. A combined model for initiation and propagation of damage under fatigue loading for cohesive interface elements. Composites: part A - Applied Science and Manufacturing, 41(12):1787-1796, 2010.

[25] P.W. Harper and S.R. Hallett. A fatigue degradation law for cohesive interface elements - development and application to composite materials. International Journal of Fatigue, 32(11):1774-1787, 2010. 
[26] L.F. Kawashita and S.R. Hallett. A crack tip tracking algorithm for cohesive interface element analysis of fatigue delamination propagation in composite materials. International Journal of Solids and Structures, 49(21):2898-2913, 2012.

[27] B. Landry and G. LaPlante. Modeling delamination growth in composites under fatigue loadings of varying amplitudes. Composites: part B - Engineering, 43(2):533-541, 2012.

[28] P. Naghipour, M. Bartsch, and H. Voggenreiter. Simulation and experimental validation of mixed mode delamination in multidirectional CF/PEEK laminates under fatigue loading. International Journal of Solids and Structures, 48(6):1070-1081, 2011.

[29] J. Fish and C. Oskay. A nonlocal multiscale fatigue model. Mechanics of Advanced Materials and Structures, 12(6):485-500, 2005.

[30] A. Shyanbhog. Investigation of debonding in adhesive joints due to fatigue. Brunel University, Uxbridge, UK, 1998. MSc Thesis. 